\title{
Frontal mucopyocele with bone erosion in a 7-year-old child - exclusively endoscopic endonasal approach - a case report*
}

Alexandre Wady Debes Felippu', Alexandre José de Sousa Cunha², Ana Carolina Silveira de Oliveira', Thiago Picolli Morsch', Manuella Pedroza Limongi', André Wady Debes Felippu', Filippo Cascio ${ }^{3}$, Alexandre Felippu Neto ${ }^{1}$
Rhinology Online, Vol 4: $108-112,2021$ http://doi.org/10.4193/RHINOL/20.090

*Received for publication:

December 8, 2020

Accepted: June 11, 2021

Published: July 9, 2021

2Department of Otorhinolaryngology, Hospital Marcílio Dias, Rio de Janeiro, RJ, Brazil

Department of Otorhinolaryngology, Papardo Hospital, Messina, Italy

\begin{abstract}
A mucocele is a benign lesion that predominantly affects the paranasal sinuses, with a slow growth rate and expansive pattern. When infected, it is known as mucopyocele. Presentation of a frontal mucopyocele can be found more frequently in adults. In contrast, it is rarely seen in children since pneumatization of the sinuses usually takes place after age 7 . This report aims to present one case of a frontal mucopyocele as an important complication of a pansinusitis in a 7-year-old child and discuss the diagnosis, surgical approach, and differentials. Endoscopic endonasal surgery is a safe approach and must be taken into consideration in these situations.
\end{abstract}

Key words: mucopyocele, frontal sinus, pediatric age, Lothrop, endoscopic endonasal surgery

\section{Introduction}

A mucocele is a benign lesion that predominantly affects the paranasal sinuses, with a slow growth rate and expansive pattern. It is a consequence of the accumulation and retention of secretion, generally caused by trauma, iatrogeny after endoscopic endonasal surgery, intense sinusitis, and polyposis ${ }^{(1-3)}$. They are most frequently found in the frontal sinus (60-65\%), followed by the ethmoidal (20-30\%), maxillary (10\%), and sphenoidal sinus (2-3\%) ${ }^{(4)}$. However, in the pediatric population it is a rare manifestation ${ }^{(5)}$, due to late pneumatization of this structure, which usually only occurs around the age of $7^{(1,6)}$. Its most prevalent complications are abscesses, osteomyelitis, orbital complications, and Pott's tumor ${ }^{(7)}$. On computed tomography (CT), in general, it manifests as a homogenous isodense image with a mucoid content that eventually provokes a remodeling of the affected sinus. Even though it is benign, due to the accumulation of secretion and mucus, the pressure can elevate, causing bone atrophy and erosion, which allows the mucocele to expand through the less resistant path ${ }^{(8)}$. Several predisposal factors were identified. They were all linked to conditions that distort the sinus' drainage. This might be the reason why its frequency is elevated in the frontal sinus.

Endoscopic functional surgery has undoubtedly assumed a central role in treating mucoceles, including those in the frontal sinus, and shows less recurrence and less complications as compared to the open technique ${ }^{(8,9)}$.

\section{Case presentation}

A female, 7-year-old, was diagnosed with sinusitis 40 days before the consultation. The patient was treated with amoxicillin for 10 days, presenting partial improvement, but continued to have headaches. Twenty days after the end of the antibiotic cycle, the patient started feeling intense pain and frontal swelling. The patient was therefore taken to the emergency room where a CT of the paranasal sinuses was performed.

The $\mathrm{CT}$ showed a frontal lesion, with bone erosion of the internal and external frontal plates (Figure 1) and a pansinusitis with maxillary and ethmoid sinus remodeling. A magnetic resonance imaging was then requested and was compatible with a frontal sinus mucocele, thickening the dura-mater of the anterior fossa. Facing this situation, a new antibiotic cycle was established with cefuroxime together with a cortisone therapy of prednisolone at 


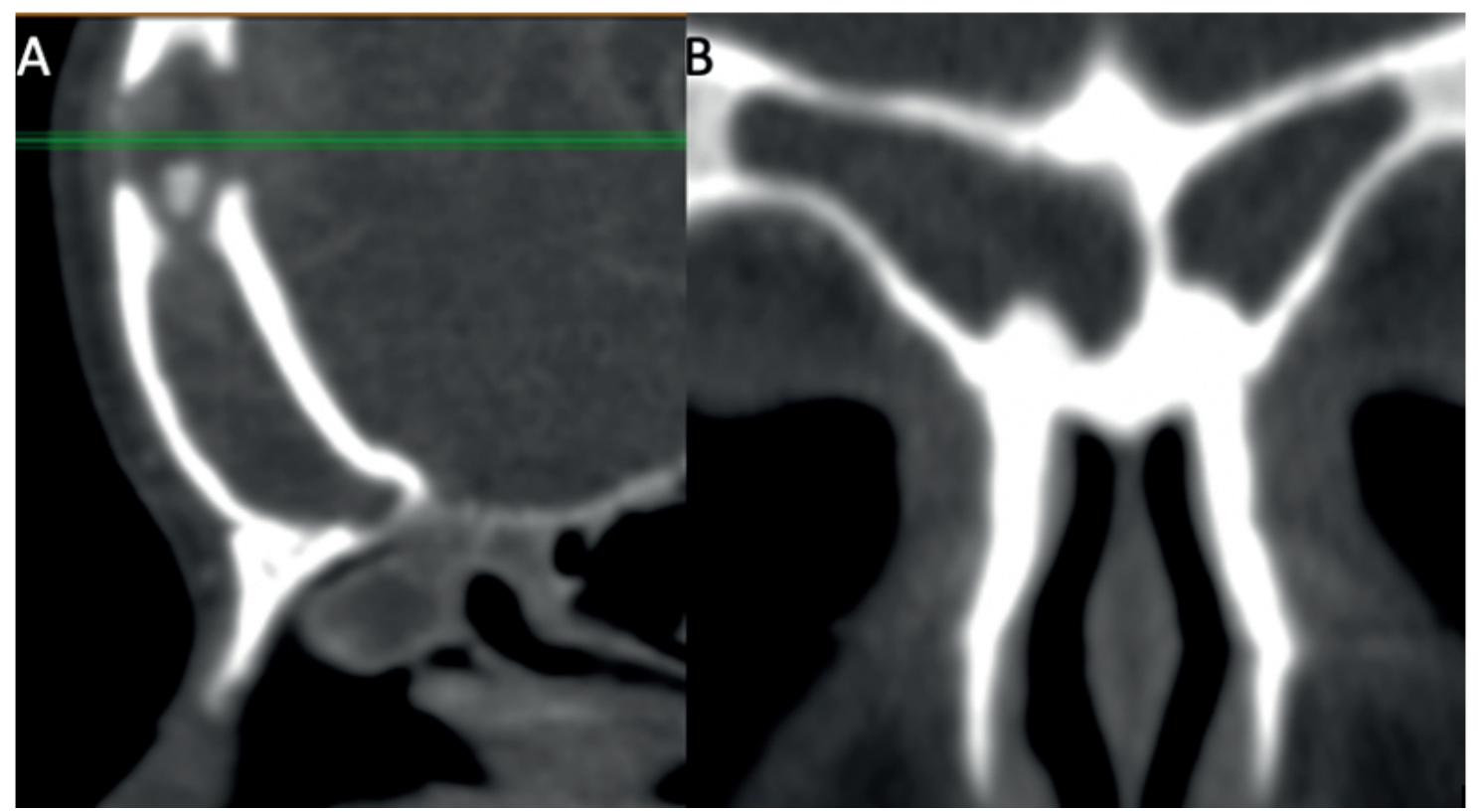

Figure 1. Preoperative CT. a) Sagittal plane, b) Coronal plane.

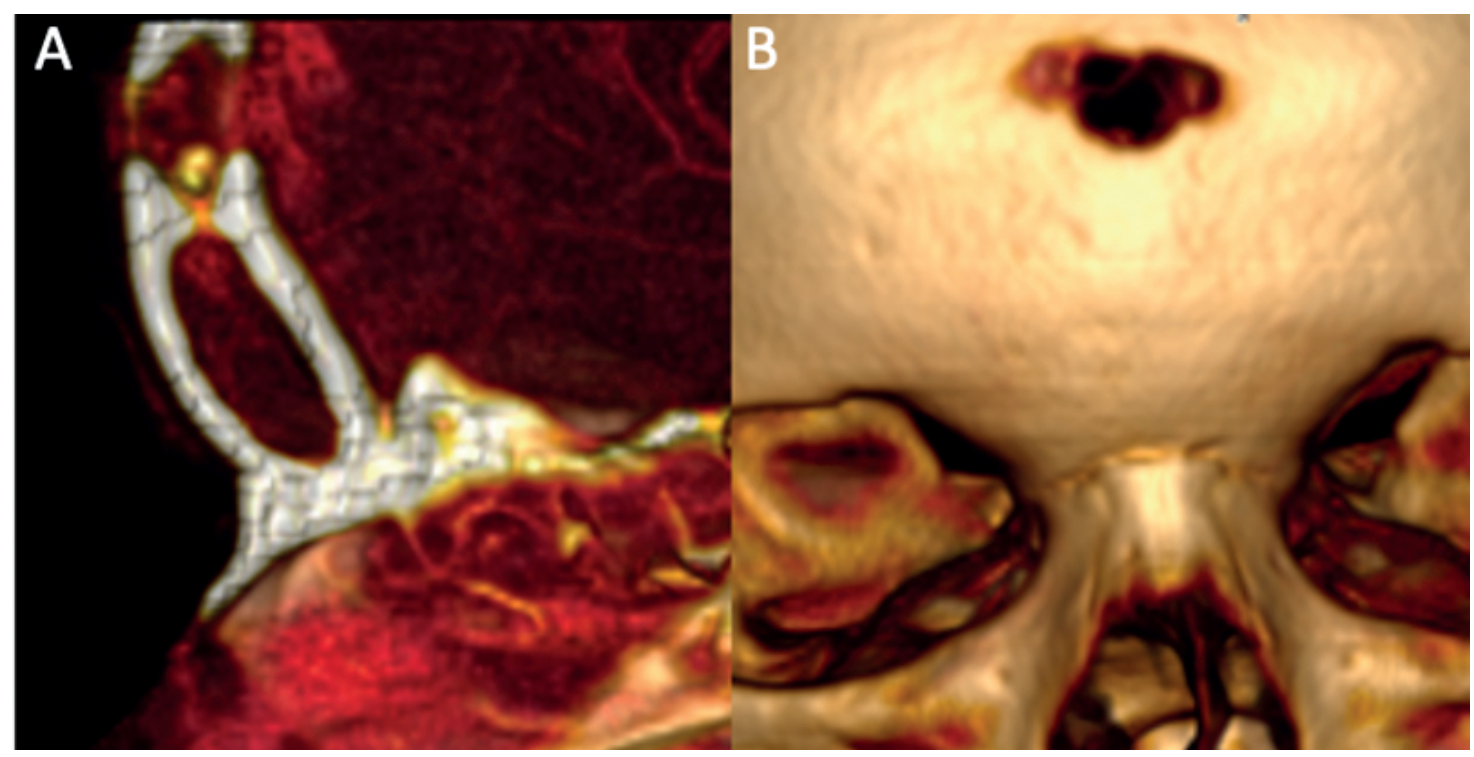

Figure 2. Preoperative 3D reconstruction. a) Sagittal plane, b) Coronal plane.

$1 \mathrm{mg} / \mathrm{kg}$ for 10 days. After the treatment, it was determined that surgery should be performed.

Surgical planning was performed with a Digital Imaging and Communications in Medicine (DICOM) viewer software to analyze all images. A 3D reconstruction (Figure 2) allowed a better understanding of the disease's behavior and, consequently, helped to define the surgical approach. This team has extensive experience with endoscopic access to the frontal sinus.

Pre-operative planning concluded that the lesion could be completely removed endoscopically. The procedure began with an endoscopic septoplasty, removing a small fragment of the superior part of the septal cartilage. After that, uncinectomy and wide antrostomies were performed, associated with a centri- petal ethmoidectomy ${ }^{(10)}$. The first fiber of the olfactory nerve was identified, followed by a transseptal access to the frontal sinus. Endoscopic modified Lothrop was executed to access the entire lesion (Figure 3) (Suppl Video 1). Although it was possible to see the entire lesion, it was extremely difficult to manipulate it. To reach the mucocele, it was necessary to "create" new surgical instruments during the operation, by reshaping flexible aspirators and curettes (Figure $3 \mathrm{~A}$ ). This played an important role in accomplishing the surgical goal (Figure 3B). A CT (Figure 4) was performed $12 \mathrm{~h}$ after the procedure for disease control, thus immediate post-operative edema can still be seen at the site of the lesion. A biopsy of the lesion was sent for analysis and its result came out as inflammatory tissue. The patient is on a 2 year 


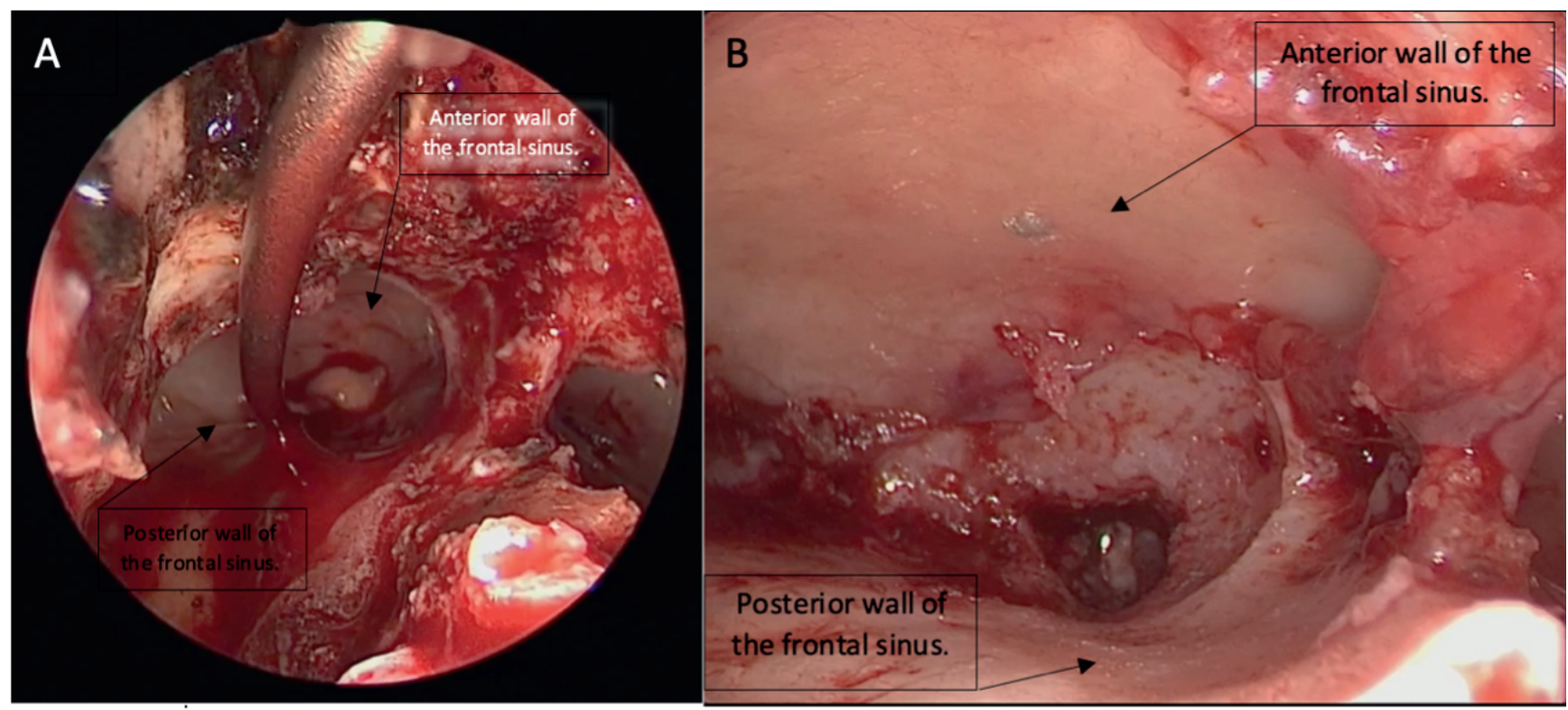

Figure 3. Intraoperative. A) Instrumentation of the lesion. Above in the figure the anterior wall of the frontal sinus can be seen. Below is the posterior wall. B) The final aspect of the frontal sinus, showing the complete overture of the lesion.

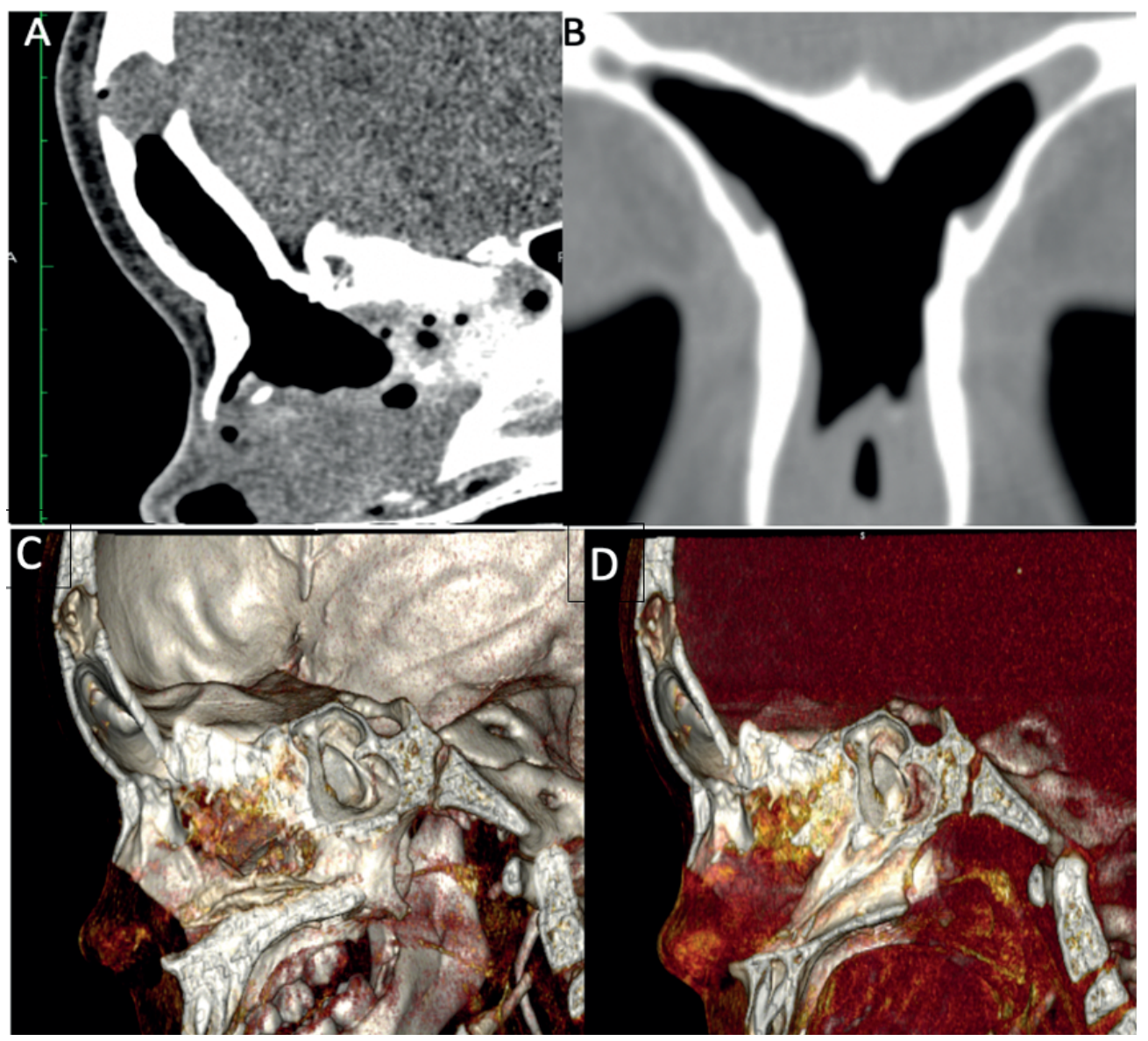

Figure 4. Postoperative CT. a) Sagittal plane, b) Coronal plane, c) Postoperative 3D reconstruction sagittal plane, d) Postoperative 3D reconstruction sagittal plane.

follow up and went through a recent telemedicine consultation. Presents asymptomatic since the procedure, with no headache, rhinorrhea or frontal swelling. Considering this clinical state, it was decided not to perform a new CT. 


\section{Discussion and conclusion}

Although frontal mucocele is often operated in a combined approach, we were able to remove the lesion with an endoscopic endonasal technique. Transseptal access to the frontal sinus allowed for good visualization of the entire lesion. Utilization of flexible instruments and an angled $\left(30^{\circ}\right)$ endoscope was essential. Ethmoidectomy by using the Centripetal technique ${ }^{(10)}$ was performed to treat sinusopathy. It also allowed a better understanding of the boundaries, since the basis of this technique relies on identification of the orbit and the skull base, which are the main anatomical structures to be dissected in order to perform a modified Lothrop. Functional endoscopic surgery has undoubtedly assumed a central role in treating mucoceles, including the ones in the frontal sinus, showing less recurrence and fewer complications than the open technique ${ }^{(8,10)}$. Moreover, this less invasive treatment may be particularly useful in children and young adults, when the incision necessary for the external approach is not always cosmetically acceptable (11). Due to the distinct topography of the lesion described in this case, pre-surgical planning was of great importance. Using a DICOM viewer software to analyze the images, it was possible to accomplish a 3D reconstruction. 3D images allowed for a better understanding of the disease's behavior, which was of great value in deciding the approach to be used.

Although frontal sinusitis in children is unusual, it is a disease that should be considered and may cause severe complications. Surgical planning is facilitated when a thorough tomographic study is first done using digital DICOM viewer tools. 3D reconstruction can give more confidence to the surgeon performing the intervention. Additionally, in cases of greater complexity, it is fundamental to foresee possible difficulties that shall be faced during the operation, especially the ones regarding material and instruments that will be used. Even when proper measures are taken, new obstacles may arise. Thus, a deep knowledge of anatomy, alongside high-level surgical training, is needed to achieve good outcomes.

The open technique is a procedure that and can be safely performed with good results. However, the endoscopic endonasal approach is a less invasive option, with lower morbidity and similar recurrence rate (close to $0 \%)^{(11-14) .}$ In consideration of a 7-year-old patient, the endoscopic endonasal approach was a good option since it allowed the removal of the disease without aesthetic damage, presented low morbidity and good recovery in the post-operative period. The case was exhaustively studied and after thorough analysis of the images (CT scan and MRI), we have concluded that it was possible and worth doing the surgery by endonasal access, even with predicted difficulties, which did occur. We could manage them, however, with the use of angled endoscopes and instruments; some of them needed to be reshaped during the surgery. This procedure was able to successfully provide removal of the mucocele. The postoperative had no complications, with no evidence of recurrence two years after the surgery. The endoscopy examination presents no signs of disease and the patient remains asymptomatic, normally developing cranio-facial structures, confirming that the procedure accomplished its goals. With the endoscopic approach it might have been more difficult to manipulate certain areas of the frontal sinus, especially in higher and lateral areas. However, this case shows that even those locations can be accessed and present excellent outcomes with low morbidity and great disease control, making it a valuable option that must be considered in similar conditions.

\section{Authorship contribution}

AWDF was the first author and participated in every task. ACSdeO, MPL, TPM and FC helped on the bibliographic revision, initial writing and translation. AWDF and AJdeSCguided the writing. AFN was the responsible for the case, guided the writing and helped with final revision.

\section{Acknowledgments}

Not applicable.

\section{Funding}

There was no funding for this report.

\section{Ethics approval and consent to participate}

The person responsible for the patient authorized publication of the case report.

\section{Consent for publication}

Written informed consent for publication of their clinical details and/or clinical images was obtained from the patient/parent/ guardian/relative of the patient. A copy of the consent form is available for review by the Editor of this journal.

\section{Availability of data and materials}

The datasets used and/or analyzed during the current study are available from the corresponding author on reasonable request.

\section{Conflict of interest}

The authors declare that they have no competing interests.

\section{List of abbreviations}

CT: computed tomography; DICOM: Digital Imaging and Communications in Medicine.

\section{References}

1. Plikaitis CM, Purzycki AR, Couture D, David
LR. Pediatric frontal mucocele secondary to a bifid frontal sinus septum. J Craniofac
Surg. 2010;21(5):1525-8.

2. Meetze K, Palmer JN, Schlosser RJ. 
Frontal Sinus Complications after Frontal Craniotomy. Laryngoscope. 2004;114(5):945-8.

3. Khong JJ, Malhotra R, Selva D, Wormald PJ. Efficacy of endoscopic sinus surgery for paranasal sinus mucocele including modified endoscopic Lothrop procedure for frontal sinus mucocele. J Laryngol Otol. 2004;118(5):352-6.

4. Lee KC, Lee NH. Comparison of clinical characteristics between primary and secondary paranasal mucoceles. Yonsei Med J. 2010;51(5):735-9.

5. Diaz F, Latchow R, Duvall AJ, Quick CA, Erickson DL. Mucoceles with intracrania and extracranial extensions. Report of two cases. J Neurosurg. 1978;48(2):284-8.

6. McCarthy JG, Karp NS, LaTrenta GS, Thorne CHM. The effect of early fronto-orbital advancement on frontal sinus development and forehead aesthetics. Plast Reconstr Surg. 1990 Dec;86(6):1078-84.

7. Borkar S, Tripathi AK, Satyarthee G, Sharma BS, Mahapatra AK. Frontal mucocele pre- senting with forehead subcutaneous mass: An unusual presentation. Turk Neurosurg. 2008;18(2):200-3.

8. Peixoto C, Andrade S, Simões M, Bastos J Ribeiro C. Mucocelo frontal: Que abordagem cirúrgica? A propósito de 2 casos clínicos. 2013;51.

9. Fisher SR. Endoscopic Sinus Surgery for Mucoceles: A Viable Alternative. Arch Otolaryngol Head Neck Surg. 1989;115(7):779.

10. Felippu A. Nasal centripetal endoscopic sinus surgery. Ann Otol Rhinol Laryngol. 2011;120(9):581-5.

11. Sciarretta V, Pasquini E, Farneti G, Ceroni AR. Endoscopic treatment of paranasal sinus mucoceles in children. Int J Pediatr Otorhinolaryngol. 2004;68(7):955-60.

12. Hartley BEJ, Lund VJ. Endoscopic drainage of pediatric paranasal sinus mucoceles. Int J Pediatr Otorhinolaryngol. 1999;50(2):10911

13. Bockmühl U, Kratzsch B, Benda K, Draf W. Surgery for paranasal sinus mucocoeles:
Efficacy of endonasal micro-endoscopic management and long-term results of 185 patients. Rhinology. 2006;44(1):62-7.

14. Nicollas R, Facon F, Sudre-Levillain I, Forman C, Roman S, Triglia JM. Pediatric paranasal sinus mucoceles: Etiologic factors, management and outcome. Int J Pediatr Otorhinolaryngol. 2006;70(5):905-8.

Alexandre Wady Debes Felippu Instituto Felippu de Otorrinolaringo-

logia

São Paulo, SP

Brazil

Tel: +55 (11) 972243575

E-mail: alex@felippu.com

\section{SUPPLEMENTAL DATA}

Video 1. Video shows the removal of the interfrontal septum with a scissel and then drilling of the anterior wall of the frontal sinus. It is possible to see the lesion located above on the right side of the right frontal sinus. After, you can see the removal of the mucocele and enlargement of the bone defect, using an angled curette around the site of the lesion to ensure the complete removal of the disease. 